\title{
3D Finite Element Analysis of the Stray Loss in Power Transformer Structure Parts*
}

\author{
Yan Li, Longnv Li, Yongteng Jing, Bo Zhang \\ Research Institute of Special Electrical Machines, Shenyang University of Technology, Shenyang, China \\ Email: lilongnv620@163.com
}

Received March, 2013

\begin{abstract}
In order to analyze the leakage magnetic field and stray loss in power transformer, leakage magnetic field and stray loss in structure parts of a power transformer are calculated by three-dimensional (3-D) non-linear time harmonic finite element method (FEM). The results show that stray loss and loss density in structure parts are large and which may lead to local overheating and affect performance of the transformer. The magnetic shields are used to reduce the stray loss and loss density of power transformer. Effects of these shields on stray loss and loss density of structure parts are discussed. The results show that stray loss and local overheating can be reduced and eliminated effectively by adding magnetic shields. It provides some references for the analysis of stray loss and optimization design in transformer.
\end{abstract}

Keywords: Finite Element Method; Stray Loss; Leakage Magnetic Field; Local Overheating; Magnetic Shields

\section{Introduction}

Power transformer is the core of energy conversion and transmission in power network, which is also the most important and expensive equipment. So it will have an essential impact of power network whether the transformer is safe, reliable and economic operation or not [1]. With the increase of capacity of the transformer, the magnetic leakage field is increasing which may enlarge the stray loss in the structure parts of power transformer. In the large power transformer, leakage magnetic field generated by the winding current will produce losses in the metal structure parts, and these losses is part of the transformer load losses, it often tend to local overheating because of its unevenly distribution. So it is quite necessary to study the magnetic leakage and stray loss deeply and accurately $[2,3]$.

In this paper, a practical power transformer model of type SZ10-50000kVA/110kV was applied to research the stray loss problem in large power transformer by using 3-D nonlinear time harmonic analysis. Detailed calculation and analysis was proceeded in order to determine the concentration of stray loss in transformer structure parts, and magnetic shields were used to reduce stray loss and prevent local overheating.

\section{3D Calculation Model and Calculation Method}

The 3-D finite element model in this paper is established

*This work was supported by NSFC, under Project 51177103 and Program for LNIRT in University (LT2011002). as shown in Figure 1. The analysis has been made with the following simplification and assumptions: 1 ) The 1/2 model of whole transformer model is established in order to reduce the computational time; 2) All field quantity sinusoidal variation with time, do not consider the highorder harmonic; 3) eddy current, circulation in winding and eddy current in iron core are being neglected.

Non-linear magnetic properties of tank, core and magnetic shields material were considered to calculate leakage magnetic field and stray loss. Magnetic shields material was disposed as anisotropic material based on "homogenization method [4,5]", anisotropic of the shield conductivity analog laminated effect. According to the continuity condition of $\mathrm{B} / \mathrm{H}$ between silicon steel sheet and air, the permeability of magnetic shields along lamination direction (y-direction) can be described as the following equation:

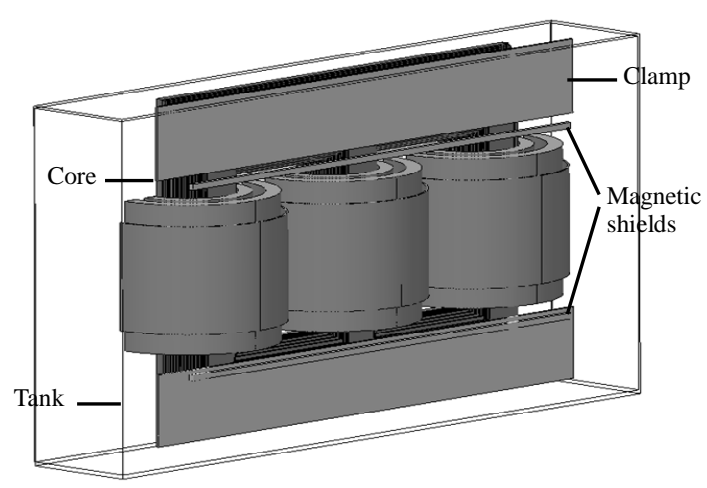

Figure 1. Structure diagram of winding. 


$$
u_{y}=u_{0} /(1-c)
$$

where $u_{y}$ is permeability of magnetic shields along lamination direction, $u_{0}$ is permeability of vacuum, $\mathrm{c}$ is lamination coefficient, taken as 0.97 . The permeability of the other two directions $u_{x}$ and $u_{z}$ are given by $\mathrm{B} / \mathrm{H}$ curve.

The eddy current generated in the silicon steel sheet near the winding side can not be ignored, the model of conductivity:

$$
\sigma=\left[\begin{array}{ccc}
\sigma_{\mathrm{x}} & 0 & 0 \\
0 & \sigma_{\mathrm{y}} & 0 \\
0 & 0 & \sigma_{\mathrm{z}}
\end{array}\right]=\left[\begin{array}{ccc}
\sigma & 0 & 0 \\
0 & c \sigma & 0 \\
0 & 0 & \sigma
\end{array}\right]
$$

In the other silicon steel sheet, the model of conductivity can be governed by following equation:

$$
\sigma=\left[\begin{array}{ccc}
\sigma_{\mathrm{x}} & 0 & 0 \\
0 & \sigma_{\mathrm{y}} & 0 \\
0 & 0 & \sigma_{\mathrm{z}}
\end{array}\right]=\left[\begin{array}{ccc}
\sigma & 0 & 0 \\
0 & 0 & 0 \\
0 & 0 & \sigma
\end{array}\right]
$$

According to Maxwell equations, transformer steady state magnetic field problem can be described as:

$$
\nabla \times \frac{1}{\mu_{e}}(\nabla \times \vec{A})=\overrightarrow{J_{s}}-\sigma \frac{\partial \vec{A}}{\partial t}
$$

where $\mu_{e}$ is permeability, $\vec{A}$ is magnetic vector potential, $\frac{e}{J_{s}}$ is current density, $\sigma$ is conductivity.

The stray loss of transformer is generally consist hysteresis loss and eddy current loss. The eddy current loss can be calculated by the following equation:

$$
p=\int_{v} \frac{J_{s} \cdot J_{s}}{\sigma} d v
$$

The average eddy current loss of time-harmonic field can be governed by following equation:

$$
p_{e}=\int_{v} \frac{\overline{\overline{J_{s}} \cdot \overline{J_{s}}}}{\sigma} d v=\int_{v} \frac{\overline{\overline{J_{0 r m s}} \cdot \overline{J_{0 r m s}}}}{\sigma} d v
$$

The hysteresis loss can be introduced in leakage magnetic field calculated on the basis of $W_{h}-B_{m}$ curve.

$$
p_{h}=\sum_{i=0}^{N} p_{h}{ }^{(i)}\left(B_{m}{ }^{(i)}\right) \sigma V^{(i)}
$$

where $p_{h}$ is hysteresis loss, $N$ is number of finite element units, $p_{h}^{(i)}$ is hysteresis loss of the $i$ unit, $B_{m}{ }^{(i)}$ is peak flux density of the $i$ unit, $\sigma$ is conductivity, $V^{(i)}$ is volume element.

The total stray loss $p$ can be governed:

$$
p=p_{e}+p_{h}
$$

\section{Verification of Calculation Method}

Leakage magnetic field and stray loss were calculated for type SFP-17000 kVA/37.6 kV practical transformer and transformer loss reference model TEAM Problem 21-B, TEAM Problem 21c-M1, TEAM Problem 21a-0 in order to confirm the calculation method effectiveness of leakage magnetic field and stray loss. The practical transformer leakage magnetic field test position diagram is shown in Figure 2. Calculation value (contain shields) and measured value of stray loss in steel plate of three models comparison results were shown in Table 1.

In Figure 2, the position II is near outer surface of $\mathrm{C}$ phase winding, from the winding center to the end. Calculation value and measured value of magnetic flux density amplitude direction component (By) comparison results was respectively shown in Figure 3(a) and (b). The loss calculation error was less than 2\% in Table 1; Calculation result and measured value was consistent in Figure 3, therefore, the loss calculation method used in the paper is effective.

\section{Analysis of Calculation Results}

\subsection{Calculation and Analysis of Loss}

In this paper, The MagNet software was using to calculate eddy current field and structure parts loss in transformer. And further, stray loss distributions in the tank wall and yoke clamp were discussed.

The loss density distribution of yoke clamp surface and tank side wall inner surface were respectively given in Figure 4 and Figure 5. Maximum loss appears in the

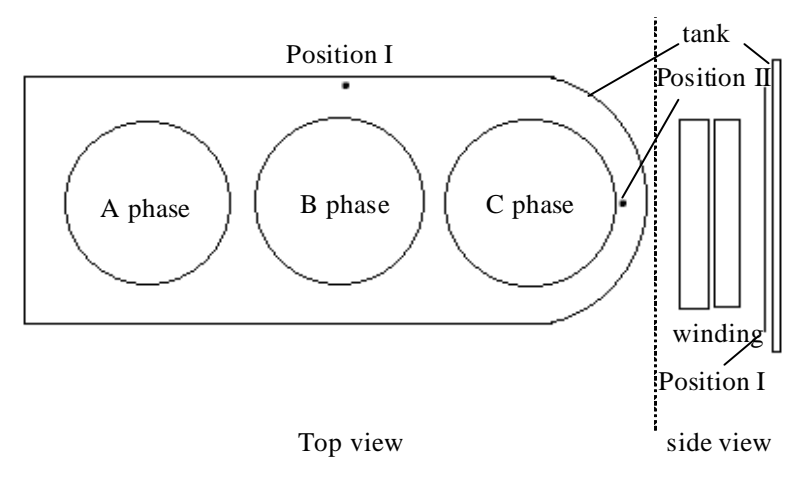

Figure 2. Test position of leakage magnetic field.

Table 1. Comparison of calculation value and measured value of loss.

\begin{tabular}{cccc}
\hline Model & $\begin{array}{c}\text { Measured } \\
\text { value/W }\end{array}$ & $\begin{array}{c}\text { Calculation } \\
\text { value/W }\end{array}$ & Error/\% \\
\hline TEAM Problem 21-B & 11.97 & 12.20 & 1.92 \\
TEAM Problem 21'-M1 & 3.72 & 3.66 & 1.6 \\
TEAM Problem 21a-0 & 9.17 & 9.24 & 0.76 \\
\hline
\end{tabular}




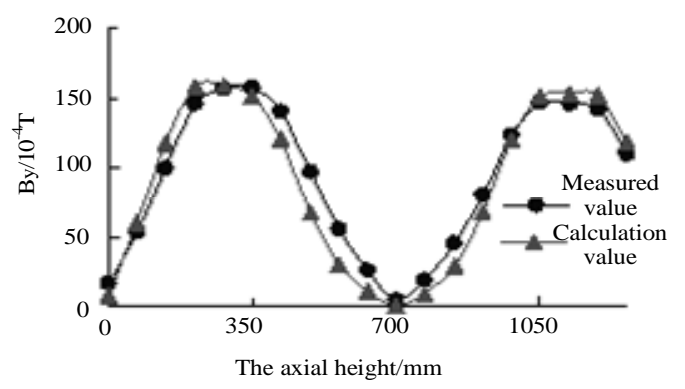

(a) Position I

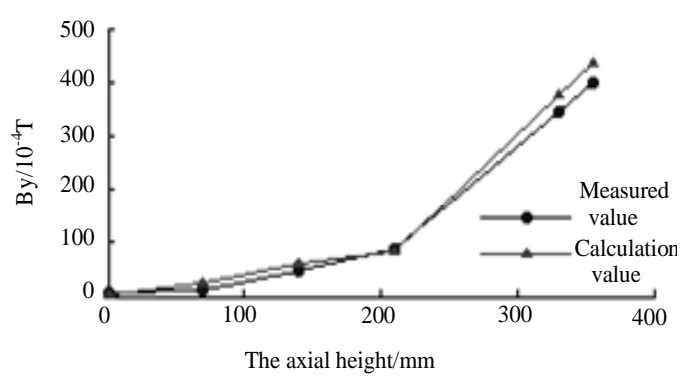

(b) Position II

Figure 3. Comparison of calculation value and measured value of magnetic flux density at assigned position.

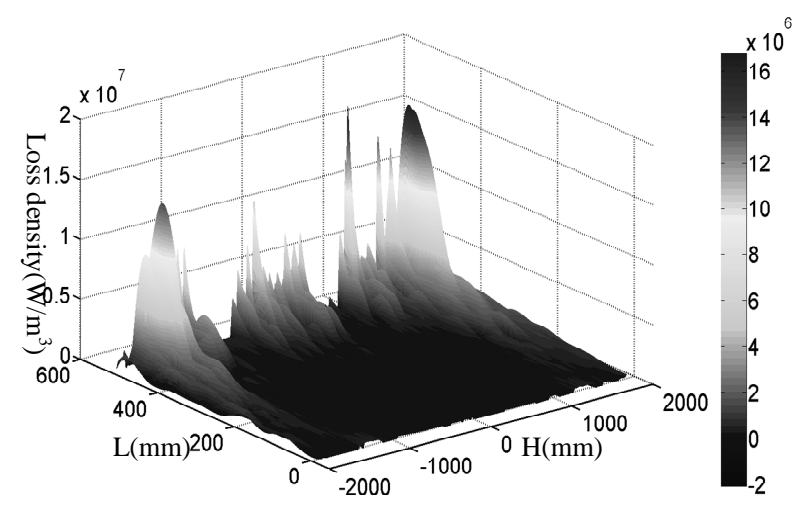

Figure 4. Diagram of loss density distribution on surface of yoke clamp.

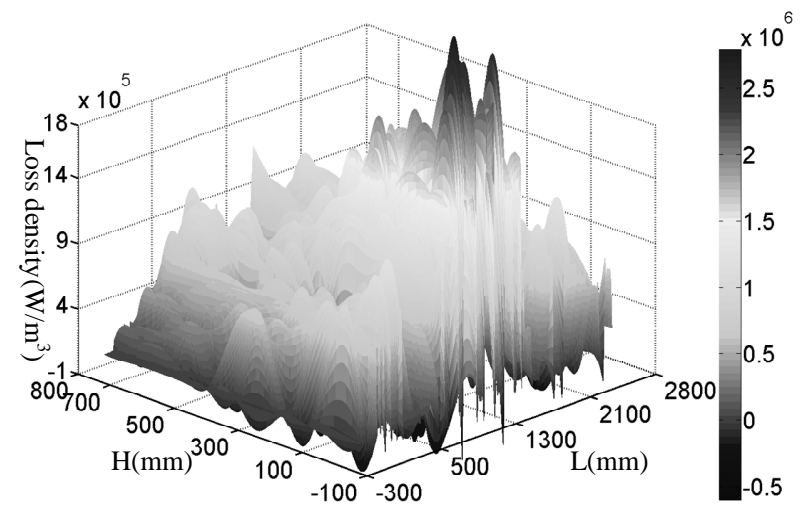

Figure 5. Diagram of loss density distribution on inner surface of tank side wall. corresponding position of $\mathrm{A}$ and $\mathrm{C}$ phase end winding, and the maximum loss density appears in the lower clamp; the loss in transformer tank was mainly concentrated in the tank side wall near $\mathrm{C}$ phase and tank wall corresponding to the middle of the three-phase windings. The $\mathrm{H}$ and $\mathrm{L}$ in diagram were respectively expressed the length and height of the tank (or clamp). The length of the tank side wall and clamp were $790 \mathrm{~mm}$ and $3760 \mathrm{~mm}$, and the heights were $2730 \mathrm{~mm}$ and $535 \mathrm{~mm}$. The stray loss and loss density of transformer tank and clamp were shown in Table 2.

\subsection{Calculation and Analysis of Loss by Adding Magnetic Shields}

The stray loss uneven distribution of transformer structure parts can cause local overheating and affect the normal performance of transformer, through adding magnetic shields can reduce the stray loss.

Magnetic shields material with high permeability attract the leakage magnetic field into the magnetic shields, prevent leakage magnetic into tank and other structure parts, thereby reduce the stray loss in the structure parts of transformer. Transformer adding magnetic shields is shown in Figure 1. Loss density of tank and clamp adding shields was decline in Figure 6 and Figure 7, maximum loss still appear near clamp and tank side wall, maximum loss density of clamp decreased by $43.1 \%$ compared to non-magnetic shields, maximum loss density of tank decreased by $11.1 \%$. The maximum stray loss and loss density of transformer structure parts with magnetic shields as shown in Table 3.

Table 2. Loss and Loss Density of Transformer Structure Parts.

\begin{tabular}{ccc}
\hline Component & Tank & Clamp \\
\hline Loss density $\left(\mathrm{W} / \mathrm{m}^{3}\right)$ & $1.8 \times 10^{6}$ & $1.6 \times 10^{7}$ \\
Eddy current loss(W) & 9846.76 & 4720.4 \\
Hysteresis loss $(\mathrm{W})$ & 3603.79 & 942.68 \\
Total loss(W) & 13450.55 & 5663.08 \\
\hline
\end{tabular}

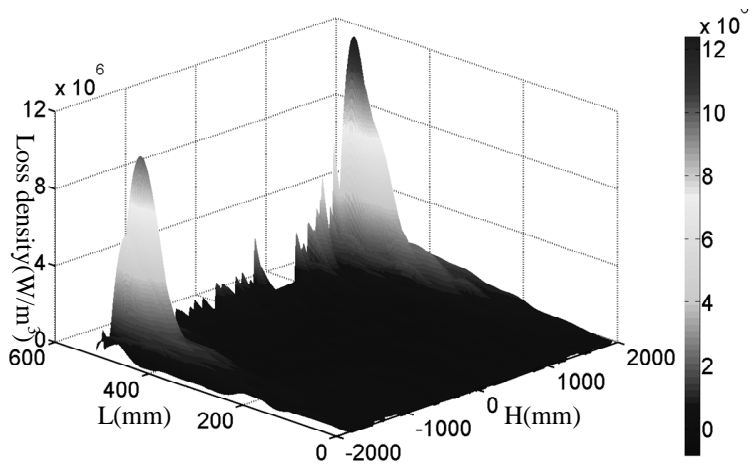

Figure 6. Diagram of loss density distribution on surface of yoke clamp with magnetic shields. 


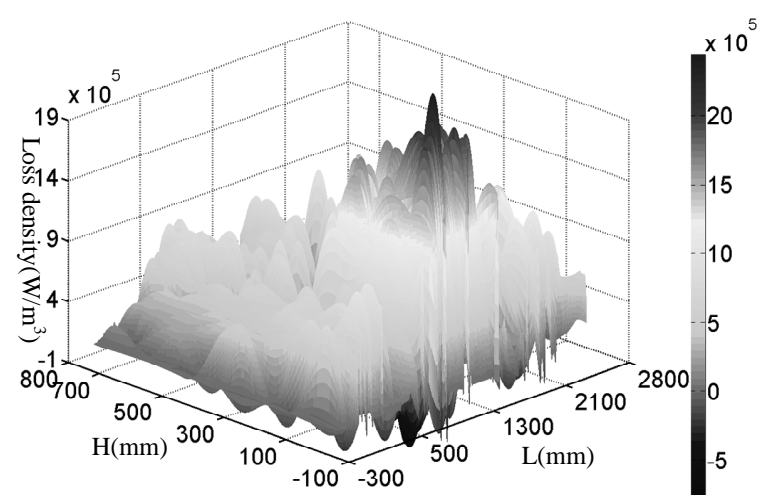

Figure 7. Diagram of loss density distribution on inner surface of tank side wall with magnetic shields.

Table 3. Loss and Loss Density of Transformer Structure Parts with Magnetic Shield.

\begin{tabular}{lcc}
\hline \multicolumn{1}{c}{ Component } & Tank & Clamp \\
\hline Loss density $\left(\mathrm{W} / \mathrm{m}^{3}\right)$ & $1.6 \times 10^{6}$ & $9.1 \times 10^{6}$ \\
Eddy current loss (W) & 5708.37 & 2313.94 \\
Hysteresis loss (W) & 2626.9 & 579.46 \\
Total loss (W) & 8335.27 & 2893.4 \\
\hline
\end{tabular}

\subsection{Magnetic Shields Effect on Structure Parts of Leakage Magnetic Field}

The magnetic flux densities of clamp surface with and without magnetic shields were shown in Figure 8 and Figure 9. Magnetic shields provide a conduction path for leakage magnetic of transformer interface winding. It can be seen from the Figure, leakage magnetic flux density significantly lower by adding magnetic shields.

\section{Conclusions}

In this paper, 3D finite element method is used to calculate the stray loss of transformer structure parts, and the conclusions are as follows:

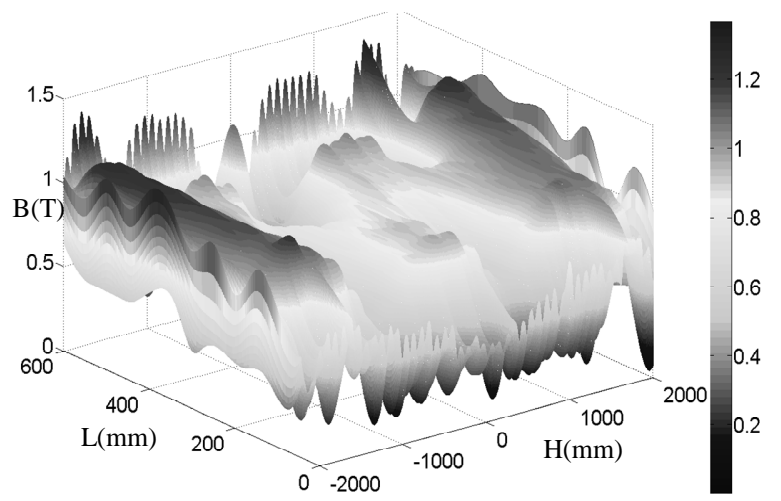

Figure 8. Diagram of magnetic flux density distribution on surface of yoke clamp with magnetic shields.

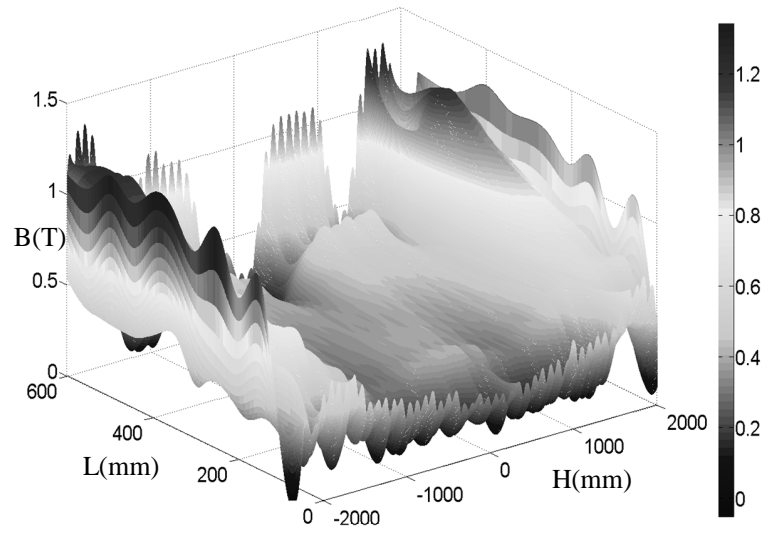

Figure 9. Diagram of magnetic flux density distribution on surface of yoke clamp without magnetic shields.

1) The results get by $3 \mathrm{D}$ finite element analysis are consistent with theoretical analysis, illustrate the validity of the method.

2) Loss and the loss density in the local place of transformer structure parts can be reduced effectively by adding magnetic shield. After adding magnetic shield to tank and yoke clamp, the maximum stray loss and loss density of tank are reduced by $38 \%$ and $11.1 \%$, the maximum stray loss and loss density of clamp are reduced by $48.9 \%$ and $43.1 \%$.

3) The density of magnetic flux leakage into the clamp is decreased obviously after adding magnetic shields.

\section{REFERENCES}

[1] M. Rizzo, A. Savini and J. Turowski, "Influence of Flux Collectors on Stray Losses in Transformers," IEEE Transactions on Magnetics, Vol. 36, No. 4, 2000, pp. 1915-1918. doi:10.1109/20.877821

[2] A. S. Reddy and M. Vijaykumar, "Hot Spot and Life Evaluation of Power Transformer Design Using Finite Element Method," Journal of Theoretical and Applied Information Technology, Vol. 4, No. 3, 2008, pp. 238-243.

[3] N. Takahashi, T. Sakura and Z. Cheng, "Nonlinear Analysis of Eddy Current and Hysteresis Losses of 3-D Stray Field Loss Model Problem 21,” IEEE Transactions on Magnetics, Vol. 37, No. 5, 2011, pp. 3672-3675. doi:10.1109/20.952687

[4] K. Laurent, D. Patrick and Z. Tarek, "Homogenization of Lamination Stacks in Linear Magneto Dynamics,” IEEE Transactions on Magnetics, Vol. 40, No. 2, 2004, pp. 912-915. doi:10.1109/TMAG.2004.825435

[5] K. Hiroyuki, K. Akihisa and F. Koji, "FEM Computation of Magnetic Field and Iron Loss in Laminated Iron Core Using Homogenization Nethod," IEEE Transactions on Magnetics, Vol. 43, No. 4, 2007, pp. 1405-1408. doi:10.1109/TMAG.2007.892429 